Ann. Biol. anim., Bioch. Biophys., Ig65, 5 (I), II3-rzo.

\title{
OBSERVATIONS SUR LA CROISSANCE DES STAPHYLOCOQUES ET LA RÉACTION LEUCOCYTAIRE AU COURS DES PREMIÈRES HEURES DE LA MAMMITE EXPÉRIMENTALE DE LA BREBIS
}

\author{
Anne LE GALL et M. PLONMET \\ Station centrale de Microbiologie et Recherches laitières, \\ Centre national de Recherches sootechniques, Jouy-en-Josas (Seinte-et-Oise)
}

SOMMAIRE

On peut suivre l'évolution de la mammite staphylococcique expérimentale de la brebis en étu. diant la multiplication bactérienne et la réaction leucocytaire in vivo par analyse d'échantillons successifs de lait.

Dans cette étude portant sur 8 I cas de mammite, on a par ce moven établi : $\mathrm{I}^{\circ}$ les caractéristiques de la multiplication bactérienne dans la mamelle; $2^{\circ}$ les conditions de déclenchement et les caractéristiques de la réaction leucocytaire.

I a réaction leucocytaire apparaît comme le principal moyen de défense contre l'infection, mais son efficacité vis-à-vis de la croissance bactérienne ne dépend ni de sa précocité ni du titre des antitoxines $\alpha$ et $\beta$. La gravité de la mammite, mesurée par l'étendue et l'intensité des lésions, est fonction du nombre maximun de germes atteint par la culture in viźo, de la quantité de lait produite par traite, des titres des antitoxines $x$ et $\beta$.

L,orsqu'on inocule une dizaine de staphylocoques dans la mamelle d'une br abis en lactation, on provoque dans les 24 heures une infection dont la gravité dépend du titre des antitoxines $\alpha$ et $\beta$ et de la quantité de lait produite par cette brebis (PLOMMET, I960; ProMmet, LE GALL, I963). L'érolution de l'infection est rapide : en 36 à 48 heures, le tableau clinique prend son caractère définitif. Ceci suppose une intense multiplication des staphylocoques et des défenses peu efficaces, au moins au cours des premières heures.

Peu de travaux ont été consacrés à la multiplication des staphylocoques in vivo, au cours de l'infection aiguë, en raison de la difficulté d'obtention de prélèvements successifs qui soient réellement représentatifs de l'évolution de la culture (FRAPPIER,

Annales de Biologie animale. - rg65. 
Sonea, I956; Gladstoxe et (irexcross I960; Foster et HuTt I960). Dans la mammite expérimentale de la brebis, par contre, on peut suivre facilement par des prélèvements successifs de lait à la fois le déreloppement bactérien in situ et la réaction leucocytaire consécutive à l'infection. Nous arons, dans ce travail portant sur $8 \mathrm{I}$ brebis, $\mathbf{I}^{0}$ établi les caractéristiques de la croissance des staphylocoques dans la mamelle au cours des $3^{6}$ heures qui suivent 1'inoculation ; $2^{\circ}$ étudié les caractères généraux de la réaction leucocytaire, en particulier le moment et les conditions de son déclenchement; $3^{\circ}$ montré que l'efficacité de la phagocytose ne dépend ni de la précocité de la leucocytose ni du titre des antitoxines $\alpha$ et $\beta ; 4^{\circ}$ montré que la gravité de l'infection dépend du titre des antitoxines $\%$ et $\beta$, de la quantité de lait sécrétée, du nombre de germes atteint par la culture.

\section{MATÉRIEL ET MÉTHODES}

8I brebis vaccinées ou non, ont été inoculées par injection de staphylocoques dans la mamelle. Les titres des anticorps résultant de la vaccination (antitosines $x$ et $\beta:$ At $x$ et At $\beta$ ) et la résistance à l'infection expérimentale ont été déja rapportés $\left(\mathrm{Ig}_{3}\right)$. De même le matériel et les méthodes décrits dans les publications précédentes $\left(1960,196_{3}\right)$ seront simplement rappelés ici.

\section{1. - Animaux}

Les brebis, de race Préalpes, étaient, au moment de l'inoculation, en lactation depuis 50 ( \pm 10$)$ jours et étaient traites, matin et soir, depuis is jours. A chaque traite, on mesurait à l'éprot:vette, la quantité de lait produite par chaque quartier $(Q:$ production moyenne du quartier par traite). Toutes les brebis étaient indennes d'infection et, à $\mathfrak{z}$ exceptions près, ne présentaient pas de séquelles d'inflammation de la mamelle.

\section{2. - Inoculation}

L'inoculation consistait à injecter par le canal du trayon dans la mamelle, préalablement vidée de son lait, $0,2 \mathrm{ml}$ d'une suspension de staphylocoques contenant entre 8 et 97 cellules. Ce nombre était déterminé par ensemencement sur gélose de 30 inoculums identiques et numération des colonies (Plommet, I960). Deux souches de staphylocoques ont été utilisées : la souche 8r-26, de type hémolytique $\alpha \beta \delta$, et, sur I 2 brebis, la souche ior-52 dérivée de la précédente, de type $\alpha \beta$. Les deux souches produisent de la coagulase libre et de la coagulase liée, mais pas de fibrinolysine. Elles appar. tiennent à un même type sérologique (j-( $\tilde{\sigma})$-16) (PiLlet et al., 1962).

\section{3. - Prélèvements}

Des échantillons de quelques $m l$ de lait ou de sérosité inflammatoire ont été obtenus par traite aseptique, d'une part immédiatement avant l'inoculation (avant la traite du soir), d'autre part $8 \mathrm{~h}, \mathrm{I} 2 \mathrm{~h}, \mathrm{I} 6 \mathrm{~h}, 20 \mathrm{~h}, 24 \mathrm{~h}$ et $3^{6} \mathrm{~h}$ après l'inoculation (avant les traites des $12^{\mathrm{e}}, 24^{\mathrm{e}}$ et $3^{6^{\mathrm{e}}}$ heures).

\section{4. - Numération des staphylocoques}

Les numérations ont été faites en ensemençant 0,1 ou $0,2 \mathrm{ml}$ de l'échantillon ou de ses dilutions décimales successives sur boîte de gélose (Tryptose Blood Agar Base, additionné de 5 p. Ioo de sang de mouton) et en incubant $2+\mathrm{h}$ à $37^{\circ} \mathrm{C}$. Lẹs échantillons prélevés $8 \mathrm{~h}$ après l'inoculation étaient 
ensemencés sur 3 ou sur 5 boîtes, purs et à la dilution-r. Les autres échantillons étaient ensemencés sur 2 boîtes par dilution. On comptait ensuite les colonies de staphylocoques, reconnais sables à l'hémolyse caractéristique qu'elles provoquent. En fait, on a presque toujours obtenı des cultures pures, sauf pour quelques-uns des échantillons prélevés à la $8 \mathrm{e}$ heure, qui contenaient un petit nombre de microcoques.
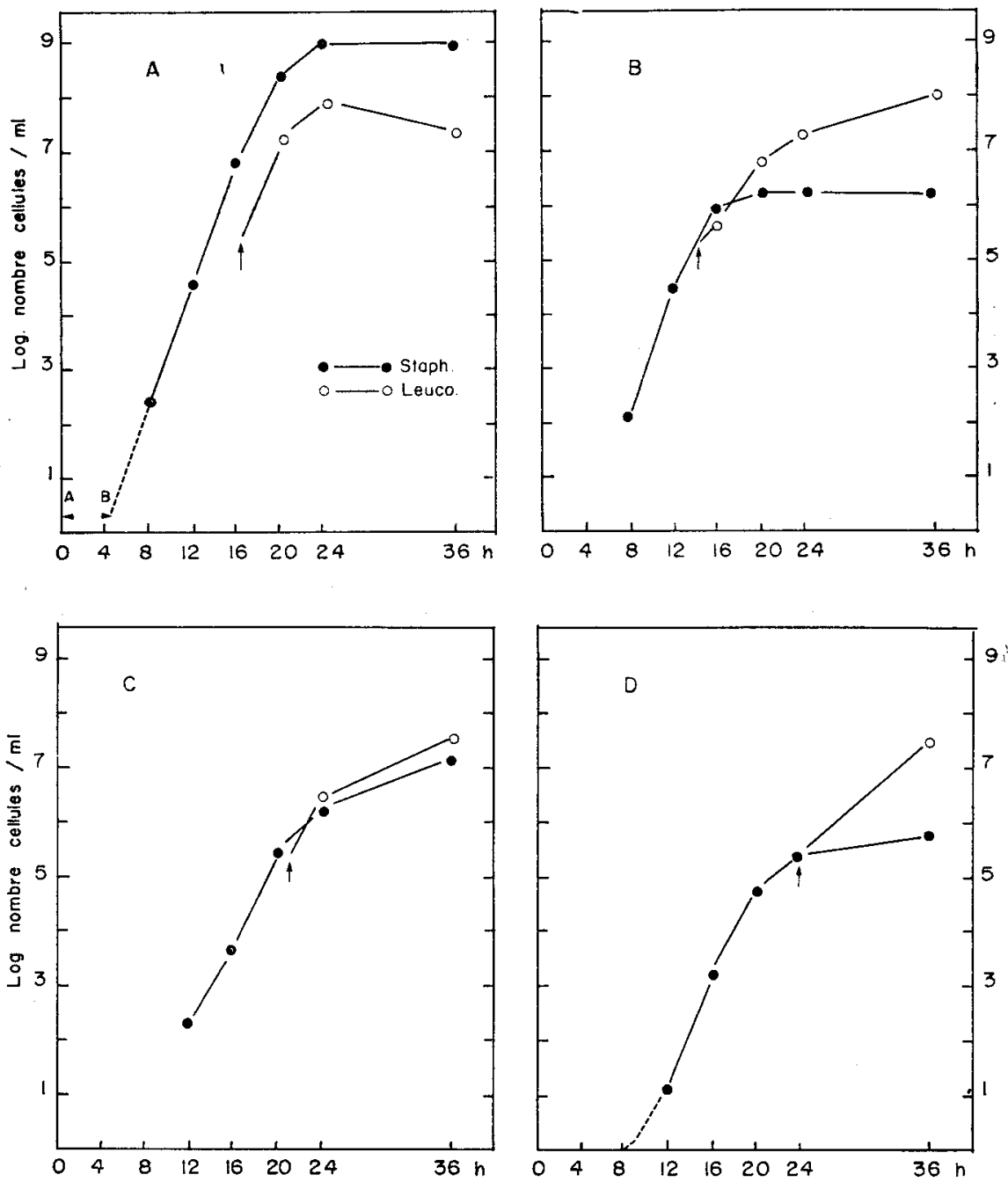

FIG. I et 2. - Croissance des staphylocoques et leucocylose dans la mammite expérimentale La flèche indique le début $R$. $\mathbf{L}$

Les échantillons des premières heures donnent régulièrement des résultats satisfaisants, les écarts entre les doubles étant inférieurs à $20 \mathrm{p}$. 100. Par contre les échantillons prélevés à la $\mathrm{i} 6^{\mathrm{e}}$ heure et au-delà donnent parfois des écarts importants entre les doubles ou les dilutions successives : : ils contiennent en effet des agglomérats de cellules et de bactéries. Pour obvier à cet inconvénient, ces échantillons ont été homogénéisés, avant numération, par agitation vigoureuse dans des tubes de dilution contenant des billes de verre. Malgré cette précaution, certaines numérations ont donné des écarts excessifs entre les doubles ; les résultats correspondants ont été considérés comme inutilisables. C'est pourquoi il manque des données correspondant à un ou plusieurs prélèvements sur certaines brebis. 


\section{5. - Numérations des cellules inflammatoires}

Les cellules du lait ont été dénombrées par la méthode de Breed, colorant de Newman, en utilisant une anse calibrée pour le prélèvement et l'étalement du lait sur la lame. Les cellules ont été comptées dans 5 à 30 champs (diamètre 0,07 ou $0,14 \mathrm{~mm}$ selon le nombre de cellules par champ) afin que les résultats soient reproductibles à $\pm 20 \mathrm{p}$. Ioo dans les limites de $1 \cdot 10^{5}$ à $1 \cdot 10^{7}$.
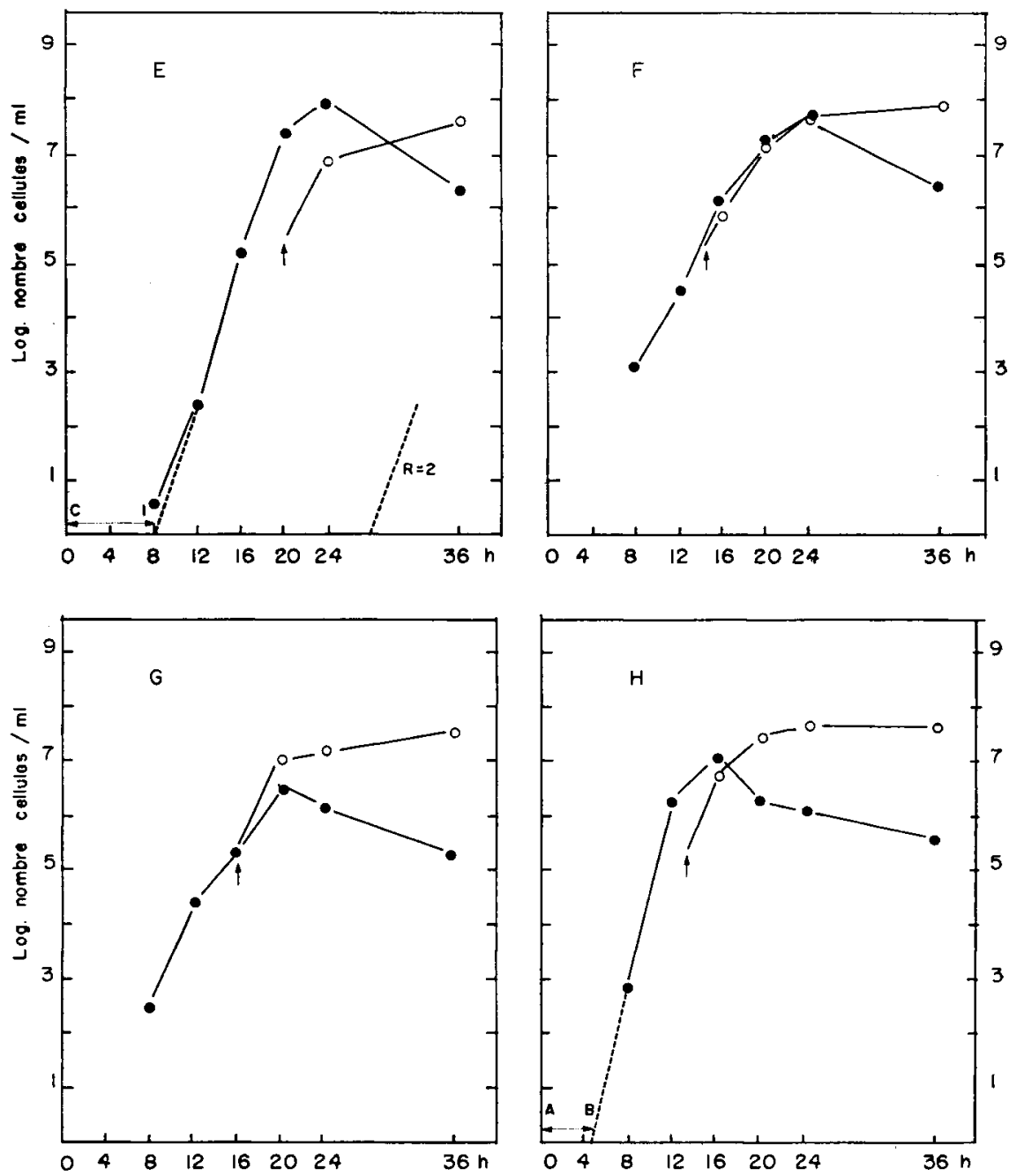

FIG. 3 et 4 . Croissance des staphylocoques ef leucocylose dans la mammite expérimentale.

Le nombre et la nature des cellules contenues dans le lait sont très caractéristiques de l'état de la mamelle : dans le lait de brebis, on trouve normalement moins de 50 ooo cellules par $\mathrm{ml}$ - cellules épithéliales surtout et quelques rares polynucléaires - ce nombre peut atteindre 200 ooo à l'occasion de petites perturbations de la lactation, au sevrage, en cas de rétention par exemple. Dans le lait des brebis guéries d'une infection, - même très ancienne --, il existe toujours un nombre plus important de cellules, jusqu'à 500000 par ml comprenant une proportion de polynucléaires comprise entre 20 et 50 p. 100 du nombre total de cellules. En période d'infection, les cellules du lait sont toujours très nombreuses et la proportion des polynucléaires atteint en général 90 à $95 \mathrm{p}$. Io0. Sur les 
courbes représentant les variations du nombre de cellules au cours de l'infection, les résultats des numérations faites avant le début de la réaction leucocytaire (R.L.) ne sont pas figurés : ainsi les courbes représentent pratiquement la variation du nombre de leucocytes. Compte tenu de ces données, nous avons défini le début de la réaction leucocytaire comme le moment où le nombre total de cellules atteint 250000 . Il a été établi avec une bonne précision à partir des courbes représentant les numérations successives (fig. I à 5 ).

\section{6. - Gravité des mammites}

Les mammites ont été classées en 6 degrés cliniçues selon l'importance de la lésion telle qu'elle apparaît à la $48^{\mathrm{e}}$ heure. Les degrés I et 2 correspondent à des mammites légères, évoluant soit vers une focalisation de l'infection ( $\mathrm{r}$ ), soit vers une extension progressive (2) ; les degrés $3 \mathrm{ct} 4$ correspondent à des mammites graves, affectant l'état général des brebis, et donnant une inflammation de la mamelle, moyenne (3) ou intense (4) ; les degrés 5 et 6 correspondent ì des mammites trì:graves, avec gangrène localisée à une partie de la mamelle (5) on affectant la totalité de l'organe (6).

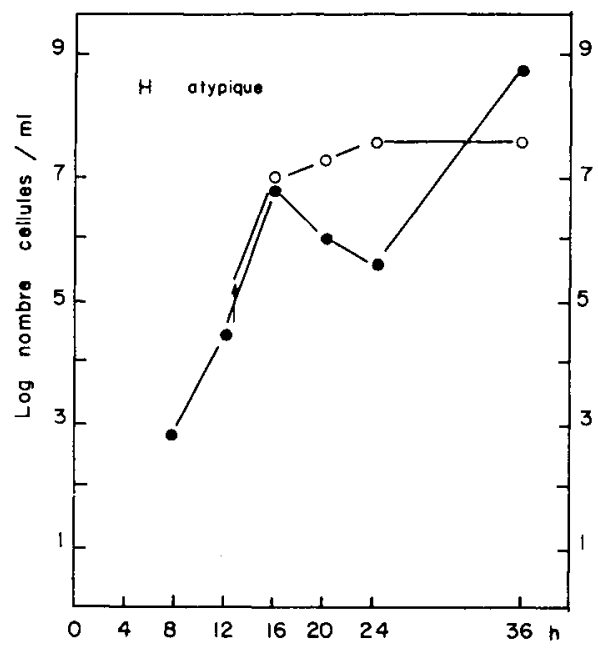

Fic. 5. Croissance des slaphylocoques et leucocylose dans la mammite expérimentale

\section{7. - Ce que représente l'échantillon}

A première vue, l'échantillon de lait semblerait représenter une culture microbienne évoluant sous un volume variable : l'inoculation ayant été faite immédiatement après une traite, le volume de la culture augmente au fur et à mesure de la sécrétion de lait jusqu'à la traite suivante, 12 heures après l'inoculation.

On doit cependant rappeler les deux faits suivants $: \mathrm{I}^{\circ}$ il reste dans la mamelle après la traite une quantité de lait de l'ordre de $20 \mathrm{p}$. roo de celle recueillie à la traite; ce lait contenu dans les acini s'écoule aussitôt vers le sinus galactophore de sorte que celui-ci, dont la capacité est de l'ordre de $25 \mathrm{p}$. 100 du volume d'une traite, se remplit très rapidement, dans les heures qui suivent la traite (Ricordeau, Martinet, Denamur, i963) ; $2^{\circ}$ la culture microbienne reste localisée, dans une première phase de l'infection, au sinus et aux gros canaux galactophores. Les échantillons de lit 8 e et de la $\mathrm{I} 2^{\mathrm{e}}$ heures représentent donc, en fait, une culture microbienne à volume sensiblement constant.

Après la traite de la $\mathrm{I}_{2} \mathrm{e}$ heure une grande partie de la culture est éliminée, puis remplacée progressivement par du milieu neuf ; la $2^{\mathrm{e}}$ phase de l'infection se développe alors, la culture s'étendant aux petits canaux, aux acini pour atteindre enfin le tissu sécrétoire lui-même.

$\mathrm{La} 2^{\mathrm{e}}$ phase se distingue cliniquement de la première par l'interruption de la sécrétion et l'apparition d'exsudat inflammatoire dans le lait. I.e passage d'une phase à l'autre a toujours lieu après la $12^{\mathbf{e}}$ heure, en général entre la $6^{\mathrm{e}}$ heure et la $20^{\mathrm{e}}$ heure après l'inoculation.

Les échantillons prélevés pendant la première phase, parce ' [u'ils viennent d'une culture en milieu liquide, sont assez représentatifs de cette culture (quelques résultats aberrants sont dus vraisemblablement à une répartition irrégulière des germes dans le sinus). Ies échantillons prélevés 
pendant la ${ }_{2} \mathbf{e}$ phase n'ont pas la même signification. L'extension progressive de l'infection, les variations de volume de la culture, l'intervention des leucocytes ne permettent pas de considérer dans tous les cas l'échantillon comme une image exacte du processus infectieux. Si, dans les mammites graves, à extension rapide, l'échantillon est encore au début assez représentatif de l'ensemble du foyer infectieux, il n'en est certainement par la suite qu'une image très imparfaite. Dans les mammites légères, l'inflammation restant localisée à une fraction de la mamelle, l'échantillon représente une dilution de la culture dans du lait normal.

\section{8. - Taux de croissance $(R)$ et temps de latence (t) apparents}

siclon les données précédentes, on peut considérer que la culture in vivo évolue à volume sensiblement constant, entre la $8^{\mathrm{e}}$ et la $\mathrm{I}_{2} \mathrm{e}$ heures. On peut par conséquent calculer à partir des numérations de $8 \mathrm{~h}\left(y_{s}\right)$, de $\mathrm{I} 2 \mathrm{~h}\left(y_{12}\right)$ et en fonction de l'inoculum $\left(y_{0}\right)$ le taux de croissance et la durée du temps de latence. Ces calculs supposent $: I^{0}$ que l'on néglige la phase intermédiaire, $2^{0}$ que la phase exponentielle soit atteinte au point $8 \mathrm{~h}, 3^{0}$ que les nombres $y_{8}$ et $y_{12}$ soient connus avec une précision suffisante.

Ln règle générale, on a admis que la condition 2 était remplie quand la valeur $y_{B}$ était supérieure à 100 puisque cela supposait une multiplication par 100 au moins de l'inoculum; on a admis aussi que $y_{8}$ et $y_{12}$ étaient connus, compte tenu des résultats des numérations, avec une précision de \pm 20 p. Ioo. I)ans ces conditions on a calculé 43 valeurs de $R$ ( $R$ calculé) et 44 valeurs de $t$ ( $t$ calculé). L'erreur sur les déterminations de $\mathrm{R}$ et de $t$ résulte $: \mathrm{I}^{0}$ de l'erreur sur les mesures de $y_{8}$ et $y_{12}, 2^{0}$ de l'erreur sur la mesure de $y_{0}$; cette dernière varie avec l'importance de l'inoculum (plus il est petit, plus l'erreur sur sa mesure est grande) et de l'estimation du volume du sinus galactophore. En prenant une erreur sur l'inoculum égale à 2 fois l'écart-type de la distribution des inoculums dénombrés sur boîte, et une erreur sur le volume du sinus égale à $t$ ro p. $100(v=0,25 \mathrm{Q} \neq 0,10 \mathrm{Q})$ on a calculé l'erreur : $\mathrm{I}^{\circ}$ sur $\mathrm{R}$ : elle est égale, en valeur absolue à $\pm 0, \mathrm{I} 5,2^{\circ}$ sur $t$ : elle est inférieure à $\mathrm{I} h$, sauf dans 6 cas se rapportant à un très petit inoculum où elle est alors comprise entre I $h$ et $2 \mathrm{~h}$.

l)ans le cas où $y_{8}<100$ germes/ml, les conditions énoncées plus haut ne sont pas remplies. Il était alors inutile de faire des calculs précis à partir de données approximatives. On ne pouvait pas, pour autant, rejeter les résultats correspondants, qui représentent justement des cas oì la phase de latence est particulièrement longue. A partir des données existantes $\left(y_{8}, y_{12}\right)$ on a fait alors une estimation graphique de $\mathrm{R}$ ( 8 valeurs); pour $t$ ( 12 valeurs), on l'a estimé en se basant sur le point $y_{12}$ et en prenant arbitrairement $\mathrm{R}=2$ afin d'éviter l'introduction d'une relation artificielle entre ces valeurs de $\mathrm{R}$ et de $t$. Les résultats correspondants sont désignés par $\mathrm{R}$ et $t$ estimés.

l.es figures I, 3 et 4 expliquent schématiquement les modes de calcul et d'estimation de $t:$ le segment $.1 \mathrm{I} 3$ représente $t$ " calculé " à partir de $\mathrm{R}$ " calculé " et de $y_{8}$ et $y_{0}$; le segment CI représente $t$ " estimé " à partir de $y_{12}$ et $y_{0}$ avec $\mathrm{R}=2$. Le fait que les calculs et estimations de $\mathrm{R}$ et de $t$ étaient basés sur un certain nombre d'hypothèses, nous a conduits ì désigner $R$ et $t$ sous le nom de taux de croissance et temps de latence apparents.

\section{9. - Mesure de l'efficacité de la phagocytose}

Pour mesurer l'efficacité de la phagocytose, plusieurs critères étaient utilisables. Il nous a semblé que le critère le plus intéressant était de mesurer l'activité globale de la phagocytose sur la croissance bactérienne. Cette activité a été estimée par la valeur de $\mathrm{R}^{\prime}$, valeur équivalente au taux de croissance (aux variations du volume de la culture près) mesurée après la R.L. R' a été calculé sur un intervalle de $+h$, le nombre de germes aux points (R.L.) et (R.L. $+4 h$ ) étant obtenu par interpolation.

\section{RÉSULTATS}

\section{I. - Croissance des staphylocoques dans la mamelle}

Compte tenu des données imprécises ou manquantes, les résultats obtenus sur $8 \mathrm{I}$ brebis inoculées ont permis de tracer 68 courbes représentant les variations du nombre de staphylocoques dans la mamelle, et de calculer ou d'estimer 5 I valeurs de $\mathrm{R}$ et 56 valeurs de $t$. Sur l'une des 3 brebis présentant des séquelles d'inflammation la croissance des staphylocoques a débuté 48 heures après l'inoculation, au-delà de la période d'observation. 
a) Caractères généraux de la croissance.

Les courbes obtenues présentent, entre elles, de grandes différences; on peut, pour schématiser, les classer en huit types principaux (fig. I à 5) selon la position du point $m$ (nombre maximal de germes) et selon la pente de la courbe au-delà de $m$.

TABLEAU I

Répartition et caractéristiques des types de courbes, selon la position du point maximum, $\mathrm{m}$, et l'écolution de la croissance au-delà

\begin{tabular}{|c|c|c|c|c|}
\hline Types & Nombre & $m(\log )$ & Heure de $m(\mathbf{1})$ & $\begin{array}{l}\text { Evolution } \\
\text { au-delà de } m\left({ }^{2}\right)\end{array}$ \\
\hline A & 9 & $s$ ì 10 & $2 x^{\prime}$ & 1) \\
\hline$B$ & 7 & b) & $16, \geqslant 0$ & 0 \\
\hline C: & ז & 7 à 10 & $3 i$ & \\
\hline $\mathrm{D}$ & $\because$ & 5 à 7 & $22_{t}^{\prime}$ & 0) \\
\hline $\mathrm{E}$ & 9 & 8 à 10 & $2^{\prime}, 20$ & $-1 ;-2 ;-3$ \\
\hline $\mathrm{F}$ & ! & 7 is & $\geq 0,2 \prime$ & $-1 ;-2$ \\
\hline$G$ & 11 & $5 \grave{a} 7$ & $16,20,2 t^{\prime}$ & $-1 ;-2 ;-3$ \\
\hline II & $13^{*}$ & $6 \dot{a} 7$ & $16,12, \geq 9$ & $-1 ;-1,5$ puis 0 \\
\hline$A E\left({ }^{3}\right)$ & 3 & $8 \dot{a} 10$ & $y^{\prime}$ & ? \\
\hline
\end{tabular}

(1) En italique : heure de m pour 1, 2 ou 3 courbes dans le groupe.

${ }^{(2)}$ Différence entre $m$ et minimum observé.

(3) Les donnces de la $36^{\mathbf{e}}$ heure, qui font la ristinction entre $A$ et $E$ manquent

(*) Une courbe atypique (voir fig. 5).

TABIEAU 2

Rípartition de la gravité des mammites en fonction des types de courbes. Grazité croissante de I à 6 (voir méthodes)

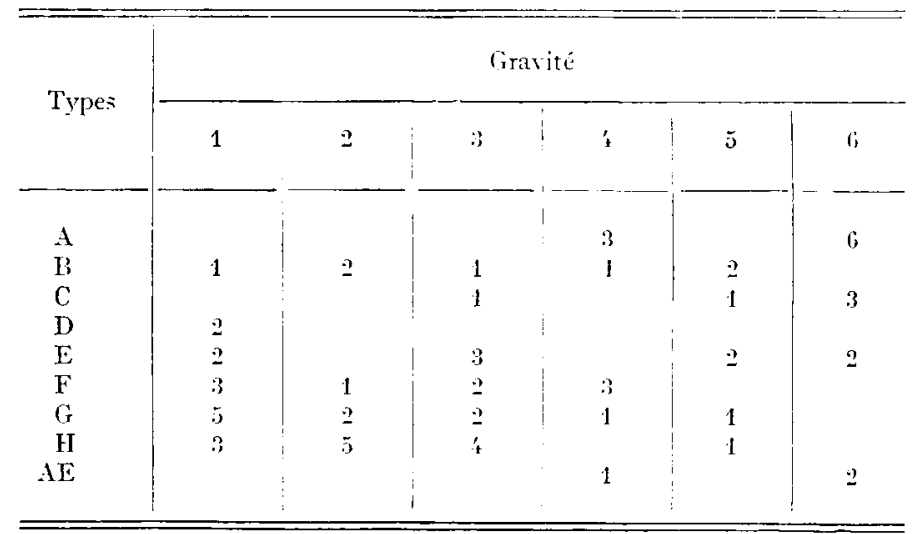


Le tableau I rapporte ces éléments ainsi que le nombre de courbes pour chaque type : $m$ est situé entre les valeurs I.IO $0^{5}$ et $6 . \mathrm{IO}^{9}$ germes $/ \mathrm{ml}$ et entre les points $\mathrm{I} 2 \mathrm{~h}$ et $36 \mathrm{~h}$. Au-delà du point $m$, les courbes présentent soit une stabilisation du nombre de germes (type $\mathrm{A}, \mathrm{B}, \mathrm{D}$ ), soit une diminution nette (types $\mathrm{E}, \mathrm{F}, \mathrm{G}, \mathrm{H}$ ) suivie elle-même d'une stabilisation à un niveau inférieur au maximum $(H)$ ou d'une nouvelle multiplication (fig. 5).

Sur certaines courbes (fig. I, 4) on peut voir une inflexion de la pente après I $2 \mathrm{~h}$ résultant de l'élimination d'une partie de la culture par la traite. Nous avons vérifié que le type de la courbe ne dépend pas de $Q$. Il y a une certaine relation entre le type de courbe et la gravité des mammites (tabl. 2).

\section{b) Temps de latence apparent (t).}

Les 56 valeurs de $t$ calculées ou estimées sont comprises entre o et Io ( \pm I heure), avec une moyenne de 3 , I heures (fig. 6). Nous n'avons pas mis en évidence de relations entre $t$ d'une part et d'autre part: $1^{0} Q$ et $y_{0} ; 2^{\circ} \mathrm{A} t \alpha$, At $\beta$ et les différentes vaccinations auxquelles les brebis avaient été soumises ; $3^{\circ} \mathrm{m}$ et le type de la courbe.

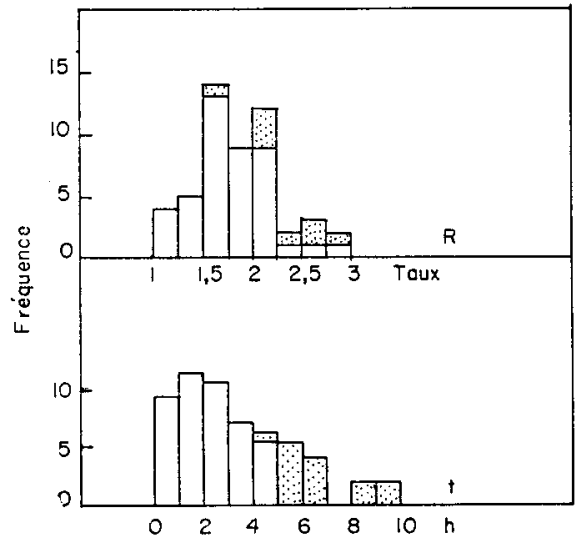

FIG. 6. - Distributions des valeurs du tan de croissance et du temps de latence apparenls (Valeurs calculées, en blanc valeurs estimées, en hachuré)

Le premier de ces trois points est conforme à ce que 1'on pouvait attendre par comparaison avec une culture in vitro. Le second point montre que les vaccinations n'apportent pas au milieu de culture des changements susceptibles de modifier le temps de latence. Le troisième point enfin, montre que l'évolution de la croissance in vivo est indépendante du temps de latence.

Il faut cependant remarquer que la variabilité de $t$, plus grande que celle qu'on obtient in vitro dans les conditions comparables, suggère l'existence d'un mécanisme d'inhibition. Le fait que deux ou trois brebis ayant des séquelles d'inflammation de la mamelle au moment de l'inoculation aient présenté des temps de latence longs $(6 \mathrm{~h})$ ou exceptionnels $(48 \mathrm{~h})$ peut faire penser qu'une phagocytose précoce n'est pas étrangère à ce phénomène; il n'y a cependant pas de relation sur l'ensemble des animaux entre $t$ et le nombre de cellules présentes dans le lait avant l'inoculation. 
c) Taux de croissance apparent $(R)$.

Les $5 \mathrm{I}$ valeurs de $R$ calculées ou estimées sont comprises entre $x$ et $3( \pm 0,15)$ (fig. 6) avec une moyenne de $I, 8$ soit un temps de génération de 33 minutes. Ces valeurs, comparables à celles que l'on peut obtenir in vitro, suggèrent que l'organisme n'oppose aucune défense efficace contre l'infection à ce stade de la croissance (avant la R.L.). Il n'y a pas de relation entre $R$ d'une part et d'autre part: $I^{\circ} Q$. Notons cependant que sur les médiocres laitières $\left(Q<5^{\circ} \mathrm{ml}\right)$, $R$ est compris entre $\mathrm{I}, 2$ et I,7 (4 valeurs) ; $2^{\circ} \mathrm{A} t \alpha$, At $\beta$ et les différentes vaccinations ; $3^{\circ} m$ et le type de la courbe.

Ces constatations, comme celles qu'expose le paragraphe précédent, montrent que $R$ ne dépend pas des facteurs contrôlés, en particulier de la vaccination et que, d'un autre côté, $R$ n'a pas d'influence sur l'érolution ultérieure de l'infection.

Entre les valeurs calculées ou estimées de $\mathrm{R}$, et de $t$, il y a une relation nette ; en particulier aux phases de latence les plus longues correspondent, en moyenne, des taux de croissance élevés, comme si une culture tardive comblait une partie de son retard par une croissance plus rapide. On peut voir, par exemple, sur la figure 6 que les valeurs estimées de $t$, qui sont toutes plus grandes que 4 , correspondent à des valeurs estimées de $R$ généralement supérieures à la moyenne. Cette relation résulte, selon toute vraisemblance, d'erreurs systématiques en plus ou en moins sur les numérations de $y_{8}$. Erreur par excès quand l'échantillon représente non pas une fraction d'une culture homogène mais la partie la plus dense, celle qui est située dans le canal du trayon, au lieu d'inoculation. Erreur par défaut, sur les valeurs de $y_{8}$ les plus basses, celles qu'on obtient par numération sans dilution, c'est-à-dire sans dissociation des agglutinats éventuels. Le premier type d'erreur doit s'appliquer à 5 ou 6 déterminations, pour lesquelles la valeur de $t$ est peu vraisemblable ( $t$ voisin de zéro). Le second type doit s'appliquer, à l'inverse, aux courbes oì $t$ est anormalement long.

\section{d) Nombre maximum de germes (m).}

Le nombre maximum de germes est compris, selon les brebis, entre I.IO $^{5}$ et 6. IO $^{9}$ germes $/ \mathrm{ml}$; il est indépendant de R et de $t$. Il n'y a pas de relations entre $m$ et $Q$ (toutefois, les brebis produisant peu de lait $(Q<50)$ ont des valeurs de $m$ inférieures à 2. $\left.\mathrm{r}^{7}\right)$. Par ailleurs, comme le montre la figure Io, $m$ est indépendant de At $\alpha$ et At $\beta$.

Les relations entre $m$ et la gravité des mammites seront étudiées plus loin.

\section{II. - Réaction leucocytaire (R.L.)}

a) Caractères généraux de la R.L.

La R.I. se traduit par l'apparition d'un nombre rapidement croissant de leucocytes (polynucléaires $90 \mathrm{p}$. IOO) dans les échantillons successifs de lait ; cette réaction est très caractéristique du début de l'inflammation. I,es polynucléaires restent prédominants au cours de l'évolution de l'infection, mais peuvent présenter des altérations plus ou moins prononcées, allant jusqu'à la lyse complète dans les cas de mammite gangréneuse ; les lymphocytes n'étant pas lysés restent alors les seules cellules dénombrables. La R.L. se produit en moyenne II,2 h après le début de la croissance.

L'examen des figures I à 5 montre que la R.L. peut être suivie : $I^{0}$ d'une inflexion de la courbe de croissance (C, F) ; $2^{\circ}$ d'un arrêt de la croissance $(B, D)$; 
$3^{\circ} d^{\prime}$ une décroissance précoce $(\mathrm{G}, \mathrm{H})$ ou tardive $(\mathrm{E}) ; 4^{\circ}$ d'aucune modification $(\mathrm{A})$. Ces différents résultats peuvent être interprétés comme la traduction d'une phagocytose plus ou moins efficace. Deux facteurs paraissaient a priori susceptibles d'avoir une certaine influence sur l'efficacité de la phagocytose : Io la précocité de la R. L. ; $2^{\circ}$ la présence et le titre des anticorps antitoxiques.

\section{b) Liaisons entre la croissance bactérienne et la R.L.}

Entre la R.L. et la croissance bactérienne, deux types de liaisons réciproques existent : la croissance induit la R.L. qui agit en retour plus ou moins efficacement sur celle-là. Nous allons analyser successivement ces deux liaisons :

Si la R.L. a lieu en moyenne $I, 2 \mathrm{~h}$ après le début de la croissance bactérienne, les écarts observés sont cependant assez grands (écart-type 2,8 ) pour que l'on puisse dire que la précocité de la R.L. est très variable selon les animaux. On pourrait donc supposer que les différents animaux répondent plus ou moins rapidement à un même stimulus représenté par une culture bactérienne en croissance.

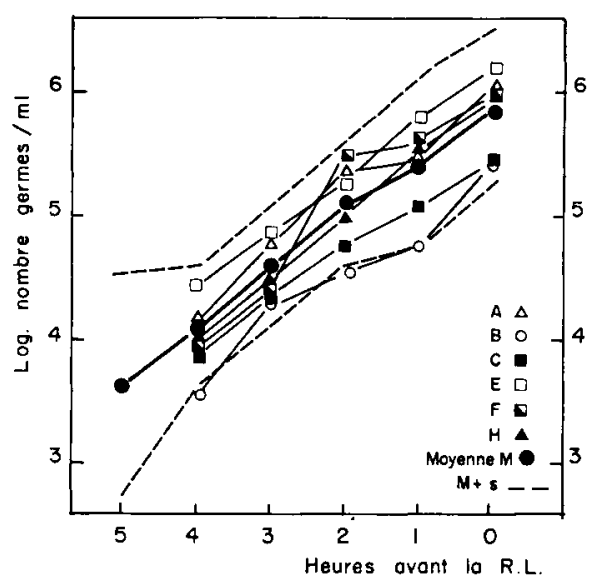

Fig. 7. - Distribution des courbes de croissance des staphylocoques par rapport all moment de la réaction cellulaire (Moyeme générale et écart-type ; moyennes par types de courbe)

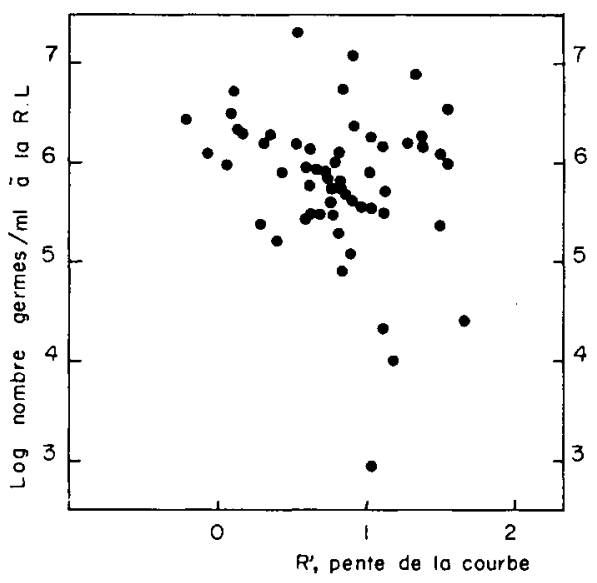

FIG. 8. - Relation entre le nombre de germes au moment le la réaction leucocytaire et la pente de la courbe (taux de croissance $R^{\prime}$ ) après celle-ci

Pour vérifier cette hypothèse on a comparé le stade de la croissance bactérienne sur les différents animaux dans les heures précédant la R.L. Pour cela, on a superposé les courbes de croissance après avoir effectué une translation telle que l'origine soit, pour toutes les courbes, le moment de la R.L. La figure 7 schénatise cette opération en représentant d'une part les courbes moyennes calculées pour chacun des types, d'autre part des courbes symbolisant la moyenne générale et la dispersion des résultats. Cette figure montre nettement ceci : pendant la période de 2 à 4 h qui préċ̀de la R.L., le nombre de germes existant à chaque instant dans le foyer infectieux diffère peu d'une brebis à l'autre. Les moyennes et écarts-types sont, en effet, pour les points $2 \mathrm{~h}, 3 \mathrm{~h}$ et $4 \mathrm{~h}$ avant la R.L. respectivement: 5, I $\pm 0,49$; $4,6 \pm 0,47 ; 4$, I0 $\pm 0,49$. Les valeurs des points situés de part et d'autre sont nettement plus dispersées; pour $o h, I h, 5 h$ avant la R.L. respectivement, ce sont: $5,89 \pm 0,62 ; 5,4 \pm 0,68 ; 3,61 \pm 0,92$. 
La conclusion de cette observation est que la R.L. est déclenchée chez toutes les brebis par un stimulus sensiblement identique, représenté par la culture en croissance à un stade donné ; on peut par exemple dire que la R.L. apparaît $3 \pm$ I heures après que la culture ait atteint le niveau de 40 ooo germes $/ \mathrm{ml}$.

Néanmoins le nombre de germes au moment de la R.L. varie dans d'assez larges limites (fig. 7 et 8 ) pour deux raisons : les taux de croissance sont assez différents d'une courbe à l'autre; la phagocytose peut débuter en fait avant le moment de la R.I. : celle-ci a été définie par un nombre de 250 ooo cellules par $\mathrm{ml}$, ce qui n'exclut pas la présence de leucocytes efficaces avant ce moment. On peut par exemple remarquer sur la figure 7 que la courbe moyenne de type $G$ (type de courbe correspondant à une phagocytose efficace) s'écarte nettement de la croissance moyenne une et deux heures avant la R.L.

Pour étudier les liaisons du $2^{\mathrm{e}}$ type, il est intéressant de considérer le rapport du nombre de staphylocoques au nombre de leucocytes à un moment donné ; on peut facilement concevoir que l'efficacité de la phagocytose puisse en dépendre. Si on choisit le moment de la R.L., le rapport a les valeurs suivantes : moyenne 3,I; moyenne \pm écart-type 0,73 et $I_{3}$; valeurs extrêmes observées 0,04 et Ioo. La figure 8 montre clairement qu'il n'y a pas de relation entre l'efficacité de la phagocytose estimée par $\mathrm{R}^{\prime}$ et le rapport précédent, exprimé par le nombre de germes au moment de la R.L.

c) Relations entre la phagocytose et les anticorps antitoxiques.

La figure 9 montre que l'efficacité de la phagocytose n'est pas en relation avec le titre de l'antitoxine $\alpha$. Cette absence de relation a été constatée de la même manière entre $R^{\prime}$ et le titre de l'antitoxine $\beta$, et avec les différents types de vaccination utilisés (corps microbiens, toxine $\delta$ ) et aussi avec $R$ et $Q$.

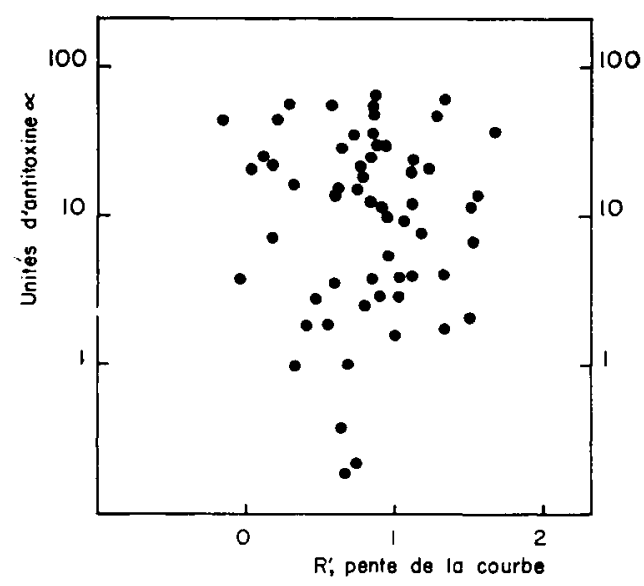

Frc. 9. - Relation entre le titre de l'antitowine et la pente de la courbe après la réaction lencocylaire

Ainsi, les différences d'efficacité de la phagocytose ne peuvent être expliquées ni par une différence de précocité de la R.I. par rapport à la croissance bactérienne, ni par une action leucotoxique éventuelle des toxines $\alpha$ et $\beta$. Les altérations des leu- 
cocytes observées au cours de l'infection ne sont d'ailleurs pas en relation avec le titre des antitoxines $\alpha$ et $\beta$. Ces altérations prouvent cependant que l'action principale des staphylocoques, à ce stade, est dirigée vers les leucocytes. L'hypothèse selon laquelle l'efficacité de la phagocytose serait sous la dépendance d'anticorps antileucotoxines non contrôlés paraît assez vraisemblable. L'observation, faite à plusieurs. reprises d'une phagocytose efficace en début d'infection, puis cédant brusquement (par exemple fig 5) s'expliquerait dans cette hypothèse par l'épuisement des anticorps intéressés.

\section{III. - Gravité de l'infection}

La gravité de l'infection, définie par l'étendue et l'intensité des lésions, résulte. de la croissance bactérienne in vivo : il faut donc s'attendre à trouver une relation directe entre la croissance et la gravité. En effet, le coefficient de corrélation entre la gravité, définie par les 6 degrés décrits, et $\log m$ est positif $\left(r_{m}=+0,593\right.$ et significatif ( $\mathrm{P} \leqslant 0$,or pour 63 données).

Nous avions déjà montré que la gravité dépend de $\mathrm{A} t \alpha$, de $\mathrm{A} t \beta$ et de $Q$. Cette dépendance pouvait être aussi être exprimée par la corrélation entre chacune de ces données prise indépendamment et la gravité. Les coefficients sont tous significatifs. $(\mathrm{P} \leqslant \mathrm{O}, \mathrm{OI})$ et ont les valeurs suivantes (données transformées en $\log (x+\mathrm{I})$ :

$$
r_{a}=-0,479, \quad r_{b}=-0,557, \quad r_{2}=+0,344 .
$$

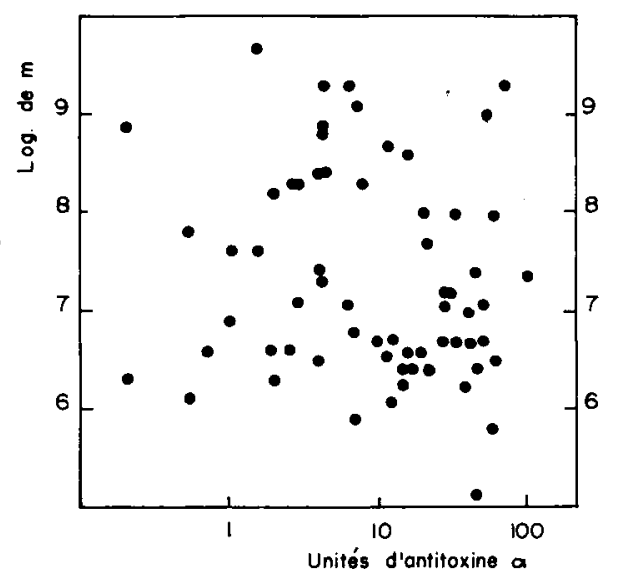

FIG. Io. -.- Relation entre le nombre maximum de germes et le litre de l'antitoxine $\alpha$

Ces variables : $Q, A t \alpha, A t \beta, m$ étant indépendantes entre elles (fig. Io), on peut cependant penser que leur association dans une équation de régression linéaire multiple est susceptible d'améliorer la prédiction de la gravité G. I1 est à noter que, si: entre At $\alpha$ et At $\beta$ il existe sur l'ensemble des valeurs une relation due au type mêmede l'expérience, les brebis vaccinées recevant simultanément les toxines $\alpha$ et $\beta$, à l'intérieur des groupes $\alpha$ et $\beta$ sont indépendants. D'autre part, s'il existe une certaine relation entre $m$ et $Q$ sur les brebis faibles laitières, ceci ne constitue que de très rares. exceptions. 
Ayant précédemment calculé l'équation de régression linéaire entre $G$ et $Q$, At $\alpha$, At $\beta$ :

$$
\hat{\mathrm{G}}=b_{1} \mathrm{Q}+b_{2} \mathrm{At} \alpha+b_{3} \mathrm{At} \beta+b_{4}
$$

nous avons vérifié ce résultat et étudié la liaison entre les résidus $(G-\hat{G})$ et la nouvelle variable $m$. Le coefficient de corrélation obtenu est de $+0,52(\mathrm{P} \leqslant 0,0 \mathrm{I})$. L'introduction de la nouvelle variable $m$ d ans une équation à 4 variables se justifie donc pleinement.

Nous donnons ci-dessous les valeurs des 2 équations de régression et des coefficients de corrélation multiple correspondants.

$$
\begin{gathered}
\mathrm{R}^{2}=0.499 \quad \hat{\mathrm{G}}=+2,94 \mathrm{Q}-0,9 \mathrm{I} \mathrm{At} \alpha-0,89 \mathrm{At} \beta-2,587 \\
\mathrm{R}^{2}=0,654 \quad \hat{\mathrm{G}}=+\mathrm{I}, 94 \mathrm{Q}-0,86 \mathrm{At} \alpha-0,67 \mathrm{At} \beta+0,69 \mathrm{~m}-5,724
\end{gathered}
$$

A titre indicatif nous avons représenté schématiquement la distribution des écarts de $(\mathrm{G}-\hat{\mathrm{G}}$ ) dans les 2 cas (fig. II). Il est clair que $m$ apporte une amélioration de la prédiction par rapport àl'équation (I) cité précédemment. Les coefficients de régression partielle de l'équation (2) sont significatifs.

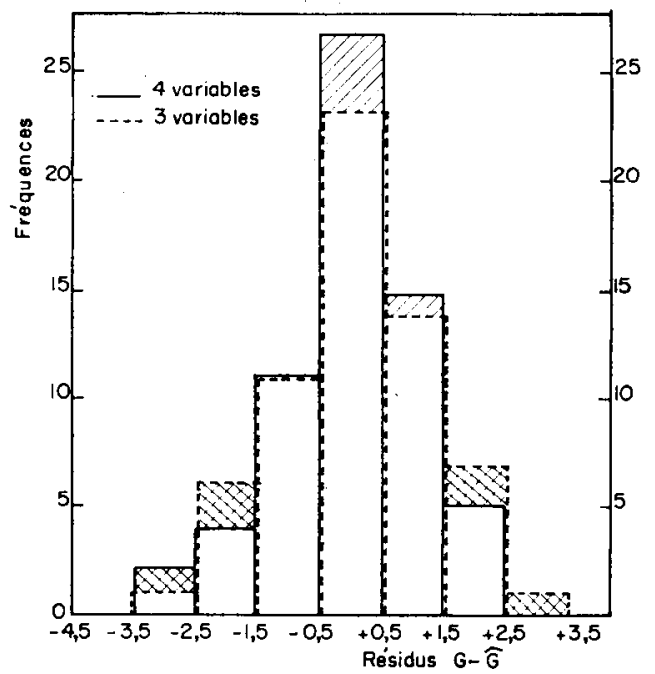

FIG. II. - Distribution des écarts de $G-\hat{G}$ pour 3 et 4 variables

Ils sont significatifs $\left(\mathrm{P} \leqslant 0,05\right.$ pour $\mathrm{Q}$ et $\mathrm{P} \leqslant 0,0$ or pour $m$, At $\alpha$ At 3 (Test $\mathrm{T}^{2}$ : respectivement 5,$874 ;$ II, $598 ; 8,0$ I $8 ; 26,067)$. On peut par conséquent faire 1'hypothèse suivante : la gravité des lésions est fonction de la quantité des toxines $\alpha$ et $\beta$ produites et non neutralisées par les antitoxines; la quantité de toxine est ellemême proportionnelle au nombre total de germes existant dans le foyer infectieux, ce nombre étant représenté par le produit $m \times Q$.

Nous admettrons qu'il ne faut pas attribuer à une analyse de ce type une valeur absolue, car elle repose au départ sur certains hypothèses et approximations ; c'est ainsi que dans l'estimation de la gravité en six degrés, il y a nécessairement un élément subjectif qui rend hasardeuse une interprétation rigoureuse et ne permet pas 
en particulier d'apprécier si les résidus sont dus à un facteur non contrôlé ou sont simplement dus à des variations aléatoires. Mais les résultats sont cependant suffisamment nets pour assurer que la gravité dépend bien des 4 éléments contrôlés pris séparément.

\section{DISCUSSION}

L'évolution de la mammite staphylococique expérimentale de la brebis a lieu en 4 phases successives : une phase de latence; une phase de croissance bactérienne sans réaction apparente de l'organisme infecté ; une phase de réaction leucocytaire ; une phase lésionnelle.

Pendant la phase de latence, les staphylocoques inoculés dans la mamelle ne se multiplient pas, au moins en apparence. Cette phase dure en moyenne $3 \mathrm{~h}$; mais elle peut quelquefois atteindre ro $h$. Elle correspond naturellement au phénomène très général de latence des cultures microbiennes repiquées dans un milieu neuf. On peut toutefois se demander, en raison de la grande variabilité observée et du retard apparent présenté par certaines cultures (décelé par le taux de croissance anormalement élevé de ces mêmes cultures), si certains mécanismes de défense n'interviennent pas au cours de cette phase. On peut imaginer des phénomènes d'agglutination, des phénomènes d'inhibition, ou des phénomènes résultant de l'intervention de leucocytes préexistants. Concernant ce dernier point, on sait déjà que la présence d'un certain nombre de leucocytes retarde considérablement le développement de l'infection et peut même l'empêcher totalement (Plommet, Ig6o; Schal,m, LasMANis, Carrol, I, I964). 'Toutefois, si de tels mécanismes existent, ils n'ont qu'un intérêt assez mince: la vaccination avec les antigènes utilisés est sans action sur eux ; les leucocytes ne sont pas normalement présents dans la mamelle saine; enfin, même en supposant que l'on puisse allonger artificiellement la phase de latence. il ne semble pas, d'après les observations de phases de latence naturellement longues, que ceci puisse modifier l'évolution ultérieure de la croissance microbienne in vivo.

Pendant la phase de croissance, d'une durée moyenne de II $h$, les germes se multiplient régulièrement sans rencontrer, semble-t-il, la moindre résistance de la part de l'organisme ; le taux de croissance moyen élevé est déjà en soi un argument ; le fait que la vaccination, qui augmente considérablement la résistance des animaux à l'infection, soit sans action sur le taux de croissance, et que l'évolution ultérieure de l'infection soit sans relation avec ce taux de croissance, sont d'autres arguments de poids. Il n'est pas sans intérêt de remarquer que les brebis les plus médiocres laitières (qui ont aussi une très petite mamelle) présentent des taux de croissance relativement bas, sans que l'on puisse donner à ce fait une explication satisfaisante.

La première réaction décelable de l'organisme, l'arrivée des leucocytes dans le foyer infectieux, caractérise le début de la $3^{\mathrm{e}}$ phase. Cette réaction a lieu $\mathrm{II}, 2 \mathrm{~h}$ (écart-type $2, S$ ) après le début de la croissance et $3 \mathrm{~h}$ (écart-type I) après que la culture ait atteint le nombre de 40 ooo germes $/ \mathrm{ml}$; la réaction leucocytaire est ainsi directement liée à la croissance bactérienne, toutes les brebis réagissant dans des délais assez voisins. Jusqu'à ce stade les animaux ne présentaient pas de différences bien nettes entre eux, qu'ils soient en définitive résistants à l'infection ou non. Par contre, dès le début de la réaction leucocytaire les différences deviennent manifestes : 
sur les animaux résistants, la croissance bactérienne est ralentie ou même arrêtée ; il $y$ a stabilisation ou diminution du nombre de germes; sur les animaux sensibles, au contraire, la croissance des staphylocoques n'est que peu ou pas modifiée, atteignant jusqu'à $6 . \mathrm{IO}^{9}$ germes par $\mathrm{ml}$ de culture. Selon toute vraisemblance, la phagocytose représente le mécanisme principal, sinon exclusif, de cette action, de sorte que la résistance à l'infection découle de l'efficacité de la phagocytose. Il est très remarquable que l'efficacité de la phagocytose (mesurée par le ralentissement de la croissance bactérienne après la R.L.) ne dépende d'aucun des facteurs contrôlés dans ces expériences, en particulier du délai de la réaction leucocytaire et du titre des antitoxines $\alpha$ et $\beta$.

Enfin, la dernière phase débute avec les premiers signes cliniques, ce qui a lieu d'autant plus tôt par rapport à la R.I. que l'infection sera plus grave : moins de $4 \mathrm{~h}$ dans les mammites de gravité 6 ; jusqu'à 24 h dans les mammites de gravité $x$. Dans le premier cas la lésion est une gangrène envahissante $48 \mathrm{~h}$ après l'inoculation; à l'opposé, elle est limitée à un petit noyau d'induration. I a gravité de l'infection, mesurée par l'importance des lésions, dépend d'une part du nombre total de germes contenus dans le foyer infectieux $(m \times Q)$ et d'autre part des quantités d'anticorps anti- $\alpha$ et anti- $\beta$ existant avant l'inoculation. Ces éléments ne sont sans doute pas les seuls ayant un rôle dans l'établissement des lésions, mais ils sont sûrement les plus importants. La relation que nous avons établie a l'avantage de regrouper en une seule formule pondérée des éléments qui, pris un à un, étaient connus pour avoir une certaine influence sur la gravité ; c'est en particulier le cas de la quantité de lait et du titre de l'antitoxine $\alpha$ (Plommes, ig6o; Plommet, Le Gali, r 963). Cette relation a aussi l'avantage de mettre en évidence le rôle de l'antitoxine $\beta$; elle souligne par là le rôle de la toxine $\beta$, qu'on avait tendance jusqu'à présent à considérer comme négligeable, à tel point que l'on n'en tenait pas toujours compte dans les travaux de recherche; la production quasi constante de la toxine $\beta$ par les souches bovines et ovines avait cependant incité les chercheurs à incorporer l'anatoxine correspondante dans les vaccins expérimentés (PILLET et al., I959; PLOMMET, LE GALI, I963). Enfin, la relation ainsi formulée fait ressortir le rôle du nombre total de germes contenus dans le foyer infectieux sur l'évolution de l'infection. Ce rôle a encore été souligné récemment par les travaux de GorRILL et MCNFIL (I963) sur les causes de mort de la souris inoculée par différentes voies.

L'analyse des phases successives de l'infection laisse entrevoir ou au contraire montre clairement certains des mécanismes de défense de l'organisme. Les premières phases ne semblent pas avoir à cet égard une grande importance ; il serait par ailleurs difficile, dans l'état actuel de nos connaissances, de mettre à profit les mécanismes en jeu pour augmenter la résistance des animaux. Tout au contraire, les mécanismes de la phase lésionnelle paraissent parfaitement clairs; il y a fort longtemps que l'on met à profit la vaccination par les anatoxines $\alpha$ et $\beta$; en améliorant les conditions de vaccination on peut espérer réduire les séquelles de l'infection à une très petite lésion. Mais il est vraisemblablement impossible - le nombre maximal de germes étant indépendant de $A t \alpha$ et $A t \beta$ - que cette vaccination puisse donner une immunité complète, telle que tnus les germes soient rapidement et entièrement détruits dès le début de la réaction leucocytaire. Les observations faites au cours du développement la réaction cellulaire, en particulier le fait qu'il y a des différences très importantes dans l'efficacité de la phagocytose, bien que sur tous les animaux la R.I. se déclare 
sensiblement au même moment de la croissance bactérienne, suggère que les leucocidines (au sens large du terme) ont un rôle primordial à ce stade de l'agression. I1 paraît vraisemblable que l'immunité complète pourrait être obtenue par vaccination contre ces substances, sans même qu'jl soit nécessaire de vacciner avec les anatoxines $\alpha$ et $\beta$, l'efficacité de la phagocytose ne dépendant pas des antitoxines correspondantes.

Le fait que la première réaction décelable de l'organisme apparaisse au moment où la culture atteint un nombre de germes de $1^{\prime}$ ordre de $I 0^{5}$ à $10^{6}$ par ml est lié naturellement au niveau du stimulus (40 000 germes $/ \mathrm{ml}$ ) et au délai (3 heures) nécessaires à sa manifestation. Ce fait explique les divergences d'opinion sur la dose minimale infectieuse: pour certains auteurs, il suffit d'un très petit nombre de germes pour déclencher une mammite expérimentale; pour d'autres, une dose élevée est nécessaire et, dans ce cas, la gravité de l'infection dépend dans une certaine mesure de cette dose (DERBYShiRE, I958). En effet, si l'on injecte un inoculum de Io ${ }^{7}$ germes, réalisant dans la mamelle une concentration de l'ordre de $I 0^{5}$ germes $/ \mathrm{ml}$, la R.L. doit se produire rapidement, en 3 heures environ; à ce moment, 1'inoculum sera resté, en raison de la longueur de la phase de latence, à un niveau très proche de $I^{5}$ germes $/ \mathrm{ml}$, équivalent à celui qui aurait été obtenu au même stade par rapport à la R.L., avec un inoculum de ro à roo germes. De plus, dans le cas d'un fort inoculum, il s'agit de germes en phase de latence, donc sensibles à la phagocytose, alors que dans 1'inoculum faible, les germes sont en croissance depuis longtemps et ont déjà produit une certaine quantité de toxines. C'est sans doute pourquoi en définitive de nombreux auteurs concluent à une dose minimale intectieuse égale ou supérieure à $10^{7}$ germes.

Notre méthode d'étude présente un certain nombre d'avantages: inoculum faible simulant une infection naturelle, résultats reproductibles et quantitatifs, prélèvements aisés, échantillons d'un volume suffisant, contrôle de la gravité de l'infection par les anatoxines $\alpha$ et $\beta$ sans modifications des premiers stades de l'infection. Cette méthode devrait permettre d'étudier avec précision les premières heures, décisives, de l'infection et en particulier d'aborder l'étude du rôle in vivo des substances ayant une action antileucocytaire. Nous ne pouvons assurer que ce type d'infection - dans un organe volumineux - peut servir de modèle aux infections staphylococciques en général, mais le rapprochement de nos résultats avec ceux de Foster et Hutr, en particulier le fait que ces auteurs observent, après inoculation intradermique à l'homme de quelques staphylocoques, des croissances telles que le phlegmon consécutif à l'inoculation contient $60.10^{6}$ germes $/ \mathrm{ml}$ après $24 \mathrm{~h}$, est assez suggestif pour permettre d'imaginer une bonne similitude des phénomènes en jeu dans ces deux types d'infection staphylococcique.

Recu pour publication en septembre I964.

\section{REMERCIEMENTS}

Nous remercions MM. J. Poly et B. Vissac et Mme L. Tassencourt du service dé Génétique de l'INRA qui ont bien voulu discuter les résultats concernant la gravité et se charger du déponillement statistique et de la réalisation des calculs sur ordinateurs. Nous remercions aussi M. J. Pillet et Mme B. OrTa de l'Institut Pasteur de Garches des discussions fructueuses que nous 
avons eues sur ce sujet. Nous remercions enfin M. G. Mocoviot, directeur de la Station centrale de Microbiologie et Recherches laitières, de l'intérêt cqu'il a porté à ce travail et de la révision du manuscrit.

\section{SUMMARY}

\section{BACTERIAL GROWTH AND LETCOCYTOSIS IN THE EXPERIMENTAL STAPHYTOCOCCAL MASTITIS IN EWES}

Lactating ewes, some of which had previously received various vaccines ( $\alpha$ and $\beta$ toxoïds, $\delta$ toxin, cells) have been given intramammary injection of 10 to 100 living cells of staphylococci. All the ewes, save one, developed mastitis, within 24 h. The cases could be classified into 6 clinical categories according to their gravity. (Frowth of bacteria and consecutive appearance of leucocytes (R.I.) were followed in the successive samples of milk at $8,12,16,20,24,3^{6}$ hours after injection, one by enumeration on agar plates and the other by direct microscopic count. 68 graphs representing the growth of staphylococci and leucocytosis were drawn. They allow to reckon: the length of apparent lag phase $(t)$; apparent maximum growth rate $(\mathrm{R})$, number of bacteria at maximum growth $(m)$, moment of the beginning of leucocytosis (R.L. : > 250000 cells per ml), number of germs before and at the R.L., apparent growth rate after R.L. ( $\left.\mathrm{R}^{\prime}\right)$.

The evolution of infection is described on four successive phases:

$\mathrm{I}^{\circ}$ lag phase, average length of which is $t=3, \mathrm{I} \mathrm{h}$ (minimum and maximum values $\mathrm{o}$ and $\mathrm{I} O$ ).

$2^{\circ}$ growth phase of bacteria without any reaction of organism, average length of which is I, $2 \mathrm{~h}$ (standart deviation 2,8 ). The average for the growth rate $R$ is $\mathrm{I}, 8$ (minimum and maximum values I,I and 2,9 ).

$3^{o}$ phase of reaction characterized by leucocytosis : R.L. starts 3 hours (standart deviation I) after the microbial growth has reached to 000 germs per $\mathrm{ml}$.

$4^{\circ}$ a clinical phase, when the lesions occur, which takes place from 4 to $2+\mathrm{h}$ after R.L. according to the gravity of infection.

The first two phases, characterized by $t$ and $R$ depend neither on inoculum, nor on quantity of milk at each milking (Q) nor on $\alpha$ and $\beta$ antitoxin titles (At $\alpha . A+\beta$ ). Existence of an inhibitor mechanism, is suggested by variability of $t$; it has no influence on subserfuent evolution of the culture.

During the reacting phase, bacterial growth is stopped, slackened or unaltered according to whether leucocytosis is efficient or not. Efficiency estimated by $\mathrm{R}^{\prime}$ and $m$ depends neither on At $\alpha$ and At $\beta$ nor on precocity of R.L. in relation with the growth of staphylococci; besides, precocity shows among the animals variations only to a small extent (log of number of germs at the R.L. : $5.8 \pm 0,62--$ standart deviation).

Intensity of lesions in the fourth phase depends signicantly on four independent factors : $m$, Q, At $\alpha$ and $A t \beta$. These two latter results emphasize part played by phagocytosis in staphylococcal mastitis and the need of control of the leucotoxic substances of staphylococci to obtain complete immunity.

\section{RÉFÉREXCES BIBLIOGRAPHIQUES}

DERbYsilne J. 13., 1958. The experimental production of Staphylococcal mastitis in the goat. J. comp. Path., 68, 232-241.

Foster W. D., IUutr II. S., I960. Experimental Staphycocial infections in man. Jancet, (2), ז373-1374.

Frappler A., Sovea S., 1956. L'immunite dans les infections staphylocociques. Action de l'antitoxine contre une infection bactérieme expérimentale. Canad. J. Microbiol., 2, 271-280.

Gladstone (i. P., Glencross E. J., I 960 . Growth and toxin production of staphylococci in cellophane sacs in vivo. Brit. J.exp. Path. 41, 31 3-33.3.

Gorrill R. H., MCNEIL E. M., I 963 . Staphylococcal infection in the mouse. The effect of route of injection. Brit. J. exp. Path., 44, +04-415.

Pillet J., Girard O., Detieil H.. Orta B., 1959. Mammite staphylococcique de la brebis et vaccination locale. Ann. Inst. Pastem, 96, $59 \mathrm{I}-600$.

Phleet J., Orta B., Perrier M., Corrieras F., Ig62. Étude sérologique de 289 souches de staphylocoques pathogénes isolées de laits de vaches et de brebis. Ann. Inst. Pasteur, 103, 7 I 6-727.

Annales de Biologie animale. - 1965. 
Plommet M., i960. Mammite staphylococcique de la brebis. Infection expérimentale. Ann. Inst. Pasteur, 98, $439-455$.

Plommet M., Le Gall A., i963. Manmite staphylococcique de la brebis. Recherches sur l'immunité antitoxique et antimicrobienne. Ann. Inst. Pastewr, 104, 779-796.

Plommet M., LE Gall A., 1963. Mammite staphylococcique de la brebis. Vaccination locale. Ann. Inst. Pasteur, 105, 535-543.

Ricorbeau G., Martixet J., Dexamer R., 1963. Traite à la machine des brebis Préalpes du Sud. Importance des différentes opérations de la traite. Ann. Z.ootechn., 12. 203-225.

Schalm O. W., Lasianis J., Carrol. E. J., I964. Effects of pre-existing Leukocytosis on Experimental Coliform (Aerobacter aerogenes) mastitis in cattle.. Amer. J. zet. Res., 25, 83-89. 